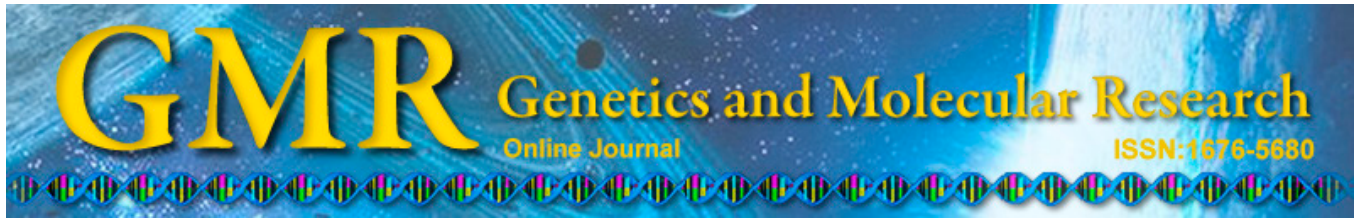

\title{
Diversity of platelet function and genetic polymorphism in clopidogrel-treated Chinese patients
}

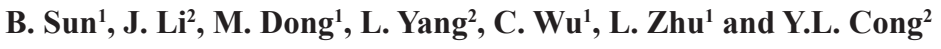 \\ ${ }^{1}$ Clinical Laboratory, The 309th Hospital of People's Liberation Army, \\ Beijing, China \\ ${ }^{2}$ Clinical Laboratory, General Hospital of People's Liberation Army, Beijing, \\ China \\ Corresponding author: Y.L. Cong \\ E-mail: ylc301@126.com
}

Genet. Mol. Res. 14 (1): 1434-1442 (2015)

Received February 3, 2014

Accepted June 10, 2014

Published February 13, 2015

DOI http://dx.doi.org/10.4238/2015.February.13.22

\begin{abstract}
We investigated the correlation between genetic polymorphisms of cytochrome P450 enzyme genes and the outcome of clopidogrel treatment in 118 coronary disease patients after percutaneous coronary intervention at the Chinese PLA General Hospital. Patients were divided into an ischemia event relapse group (IERG) and a nonIERG group (NIERG) based on relapse of ischemia events within 6 months after percutaneous coronary intervention. Ischemia occurred in $26.27 \%$ of patients. Thromboelastogram platelet mapping results showed that compared with the NIERG, the ADP-induced platelet inhibition ratio in the IERG was significantly lower $(31.33 \pm 24.91 \%$ vs $54.68 \pm 26.63 \%, \mathrm{P}<0.05)$. The platelet inhibition ratio of patients carrying mutant alleles $C Y P 3 A 5 * 3(41.98 \pm 29.33 \%$ vs $52.89 \pm 26.49 \%)$, CYP2C19*2 $(43.15 \pm 27.97 \%$ vs $55.89 \pm 26.71 \%)$, and P2Y12*1 $(38.74 \pm 24.36 \%$ vs $52.19 \pm 28.58 \%)$ was lower than patients with the wild-type alleles. The frequency of ischemia event relapse in patients
\end{abstract}


with the mutant alleles $C Y P 3 A 5 * 3$ and $C Y P 2 C 19 * 2$ was significantly higher than patients carrying the G/G genotype; however, there was no significant difference between patients carrying the $\mathrm{T} / \mathrm{T}$ genotype and $\mathrm{C}$ allele of $P 2 Y 12 * 1$. Thus, coexisting polymorphisms of $C Y P 3 A 5 * 3$ and $2 C 19 * 2$, but not $P 2 Y 12 * 1$, play an important role in the variability of clopidogrel's curative effect.

Key words: Coronary heart disease; Genetic polymorphisms; Clopidogrel; Percutaneous coronary intervention; Platelet mapping

\section{INTRODUCTION}

Clopidogrel, a second-generation thienopyridine that inhibits platelet aggregation, is a first-line treatment along with aspirin in the management of patients with coronary artery disease, acute coronary syndromes, and/or after percutaneous coronary intervention (PCI). However, a significant proportion of patients remain at risk for subsequent death, myocardial infarction, stent thrombosis, and stroke because of insufficient clopidogrelinduced platelet inhibition (Gutliikonda et al., 2005; Wang et al., 2006; Angiolillo et al., 2007; Sugunaraj et al., 2010). It has been estimated that in 4-30\% of patients, expected antiplatelet effects are absent after clopidogrel administration (Nguyen et al., 2005; Barsky and Arora, 2006). A multitude of patient-related (obesity, diabetes mellitus, and smoking) and drug-related (pharmacodynamic and pharmacokinetic) factors have been implicated for the variant response observed with clopidogrel treatment (Angiolillo et al., 2007; Camilleri et al., 2011).

Clopidogrel is an inactive prodrug that requires hepatic bioactivation via several cytochrome P450 enzymes, including CYP2C19, CYP1A2, CYP2B6, CYP2C19, CYP2C9, and CYP3A4/5 (Fitzgerald and Maree, 2007). The active metabolite irreversibly inhibits the platelet ADP receptor, P2Y12 (Fitzgerald and Maree, 2007). P2Y12 is polymorphic; the T744C variant is in linkage disequilibrium (Angiolillo et al., 2005). It exists in 2 different functional haplotypes, $\mathrm{H} 1$ and $\mathrm{H} 2$, and the latter is the rarer version of the 2 and shows more potent functional aggregation in response to ADP (Fontana et al., 2003).

CYP2C19 is polymorphic, in which the loss-of-function allele, CYP2C19*2 in exon 5 , is the most commonly observed variant. In comparison, the loss of 1 allele results in partially compromised platelet aggregation post-clopidogrel treatment (Mega et al., 2009; Chen et al., 2010; Jeong et al., 2010; Pettersen et al., 2011). Another form, CYP2C19*17, has been implicated in bleeding following clopidogrel treatment (Sibbing et al., 2010). CYP $3 A 5$ is also expressed polymorphically, among which only $C Y P 3 A 5^{*} 3$ is in linkage disequilibrium (Kuehl et al., 2001; Balram et al., 2003; Evans and McLeod, 2003; Xie et al., 2004). Notably, the wild-type $C Y P 3 A 5^{*} 1$ allele dictates enzyme activity and is present under either homo- or heterozygous conditions.

Previous studies have indicated that polymorphisms at either of the $C Y P 3 A 4 / 5, C Y$ $P 2 C 10$, or $P 2 Y 12$ loci impact and influence clopidogrel therapy efficacy in Caucasians and Asians (Fontana et al., 2003; Pettersen et al., 2011). Therefore, in the current study, we examined the singular and multivariate effects of the CYP3A5 $(22893 \mathrm{G}>\mathrm{A})$, CYP2C19 $(681 \mathrm{G}>\mathrm{A})$, and P2Y12 (744T $>$ C) polymorphisms on clopidogrel treatment outcome. 


\section{MATERIAL AND METHODS}

\section{Patients}

All study subjects gave written informed consent for participation in the study, which was approved by the Ethics Committee of People's Liberation Army General Hospital, Beijing, China. Between November 2010 and March 2011, 118 coronary artery disease patients at the Heart Institute, Department of Cardiology Care Unit, People's Liberation Army General Hospital, Beijing, China were enrolled in the study.

The 2007 American College of Cardiology/American Heart Association chronic stable angina pectoris guidelines (Tantry et al., 2005), 2008 American College of Cardiology and American Heart Association acute myocardial infarction treatment guidelines (Bliden et al., 2005), and 2011 American College of Cardiology and American Heart Association unstable angina and non-ST-segment elevation myocardial infarction treatment guidelines (Gurbel et al., 2005a) were used for diagnosis. Inclusion criteria were the need to administer clopidogrel after PCI in patients with coronary artery disease. Patients with co-morbidities such as hypertension and diabetes mellitus were not excluded.

Exclusion criteria were: 1) allergic to clopidogrel; 2) platelet count $<100 \times 10^{9} / \mathrm{L}$ or platelet count $>450 \times 10^{9} / \mathrm{L}$ and hemoglobin $<80 \mathrm{~g} / \mathrm{L} ; 3$ ) severely abnormal liver and kidney function (alanine aminotransferase/aspartate aminotransferase 3 times higher than the upper limit of normal; blood urea nitrogen $\geq 20 \mathrm{mM}$ or creatinine $\geq 445 \mu \mathrm{M}$ ); 4) severe uncontrolled hypertension (blood pressure $\geq 180 / 110 \mathrm{mmHg}$ ); 5) exposed to infections in the past week; and 6) trauma, surgery, and bleeding after January 2010.

\section{Medications}

Patients admitted were prescribed a $75 \mathrm{mg} /$ day clopidogrel dose, while PCI patients were given a $300 \mathrm{mg}$ clopidogrel loading dose after a $75 \mathrm{mg} /$ day initial maintenance dose over the same duration along with $100 \mathrm{mg} /$ day aspirin.

\section{Blood sampling}

Blood samples were collected in sodium citrate anticoagulant tubes $(0.109 \mathrm{M}, 0.3$ $\mathrm{mL}$ ) (BD Biosciences, Franklin Lakes, NJ, USA) 1-3 days after PCI and were immediately processed for platelet aggregation and hematology assays.

\section{Platelet aggregation}

Thromboelastogram platelet mapping was performed using the TEG 5000 Thrombelastograph Hemostasis Analyzer System (Haemonetics, Braintree, MA, USA) according to manufacturer instructions. The rate of inhibition of platelet aggregation was determined based on the ability of a blood clots agglutination force-generated curve to determine the function of platelet aggregation, which was the aggregation force of the maximum amplitude (MA) of the agglutination curve. 


\section{Definition of clopidogrel responsiveness}

Arachidonic acid (AA) or ADP-induced platelet inhibition was calculated using the following formula: platelet inhibition rate $(\%)=\left[1-\left(\mathrm{MA}_{\text {prothrombin }}-\mathrm{MA}_{\text {fibrinogen }}\right) /\left(\mathrm{MA}_{\text {thrombin }}\right.\right.$ $\left.\left.\mathrm{MA}_{\text {fibrinogen }}\right)\right]$ x $100 \%$. Responses included either poor aspirin responders induced by $1 \mathrm{mM}$ $\mathrm{AA}$, showing a platelet aggregation inhibition rate of $\leq 50 \%$, and a low clopidogrel response induced by $2 \mu \mathrm{M}$ ADP, showing platelet aggregation inhibition of $<30 \%$.

\section{Experimental grouping}

Samples were grouped according to the thromboelastography (TEG) inhibition of platelet aggregation rate, divided into low anti-platelet drug reactions (including low clopidogrel response and low aspirin reaction groups) and anti-platelet drug reactions in the normal group. All patients were followed up for 6 months to determine the occurrence of adverse ischemic events (in-stent restenosis, unstable angina requiring re-hospitalization, stroke, fatal or nonfatal myocardial infarction, acute or sub-acute thrombosis). All 118 patients were placed into the ischemic event recurrence group (IERG) or non-ischemic event recurrence group (NIERG).

\section{Genotyping}

Genomic DNA was extracted from blood samples post-TEG testing using a DNA extraction kit as per manufacturer recommendation (Shanghai Biological Engineering Technology Service, Shanghai, China). Polymerase chain reaction was performed using reagents obtained from Beijing Sport Century Biotech (Beijing, China). The following primers were used for genotyping: $C Y P 3 A 5 * 3$ (22893G>A): FP: 5'-GGCATAGGAGATACCCA-3'; RP: 5'-GGT TCTAGTTCATTAGGGTG-3'; GTTCTTTTACTTTCTCCAA-3'; P2Y12*1 (744T>C): FP: 5'-ATATCTTTTACACGAAAG-3'; RP: 5'-ATTACCACAATAGGCAG-3'.

Polymerase chain reaction products were resolved using agarose gel electrophoresis and analyzed using the AlphalmagerTM 2000 gel imaging analysis system (San Leandro, CA, USA). Amplification products were sequence-verified at the Beijing Sino-US Taihe Biotechnology Company (Beijing, China).

\section{Statistical analysis}

Continuous variables are reported as means \pm standard deviation, whereas categorical variables are represented as percentages. Normally distributed measurement data between the 2 groups were compared using one way analysis of variance, independent samples, Student $t$-test, skewed distribution of measurement data to the median (P25 of P75) (expressed by the conversion to approximate a normal distribution using the Student $t$-test or analysis of variance, other non-parametric methods of analysis). Count data between the 2 groups were compared using the chi-squared test. $\mathrm{P}<0.05$ was considered to be statistically significant.

\section{RESULTS}

\section{Patient group comparisons}

Of the 118 cases of coronary heart disease, 42 cases (35.59\%) showed a low antiplatelet 
drug response (low antiplatelet drug reactions group). Among them, the ADP-induced platelet aggregation inhibition rate was less than $30 \%(48.55 \pm 28.05 \%)$ in 35 cases, accounting for $29.66 \%$ of all patients in the low clopidogrel response group. The AA-induced platelet aggregation inhibition rate was less than $50 \%(75.98 \pm 27.54 \%)$ (aspirin reaction low group), accounting for $16.1 \%$ of all enrolled patients. The response to both clopidogrel and aspirin was lower in 12 cases compared to the response in 23 and 7 cases for clopidogrel and aspirin alone, respectively. There was no significant difference in patient characteristics or response to antiplatelet treatment regimens $(\mathrm{P}>0.05)$, except that older women had a higher chance of performing as low responders following clopidogrel treatment (Table 1). Red blood corpuscles and hemoglobin levels were significantly lower in the low responder group $(\mathrm{P}=0.0473$ and 0.0110 , respectively). Other hematologic and biochemical parameters showed no significant differences $(\mathrm{P}>0.05)$ (Table 1).

Table 1. Demographic, clinical, procedural and laboratory information of the enrolled coronary heart disease patients $(\mathrm{N}=118)$.

\begin{tabular}{|c|c|c|c|}
\hline & Low responders & Normal responders & $P$ value \\
\hline \multicolumn{4}{|l|}{ General } \\
\hline Gender (female) & $12(28.57 \%)$ & $10(13.16 \%)$ & 0.0396 \\
\hline Age (years) & $61.83 \pm 12.75$ & $56.13 \pm 11.27$ & 0.0178 \\
\hline BMI $\left(\mathrm{kg} / \mathrm{m}^{2}\right)$ & $25.48 \pm 3.28$ & $25.95 \pm 3.69$ & 0.4804 \\
\hline Systolic blood pressure at admission & $129.86 \pm 21.40$ & $133.43 \pm 19.44$ & 0.3270 \\
\hline Diastolic blood pressure at admission & $73.45 \pm 11.60$ & $75.50 \pm 10.92$ & 0.3513 \\
\hline Pulse pressure $(\mathrm{mM} / \mathrm{Hg})$ & $56.95 \pm 17.94$ & $57.80 \pm 17.08$ & 0.8027 \\
\hline Pressure index & $0.43 \pm 0.08$ & $0.43 \pm 0.09$ & 0.8034 \\
\hline Pulse at admission (beats/min) & $76.00 \pm 9.11$ & $77.04 \pm 8.92$ & 0.5515 \\
\hline \multicolumn{4}{|l|}{ History } \\
\hline Hypertension & $20(47.62 \%)$ & $51(67.11 \%)$ & 0.0747 \\
\hline Diabetes & $8(19.05 \%)$ & $22(28.95 \%)$ & 0.1086 \\
\hline Smoking & $24(57.14 \%)$ & $39(51.32 \%)$ & 0.5435 \\
\hline Hyperlipidemia & $17(40.48 \%)$ & $25(32.89 \%)$ & 0.2024 \\
\hline \multicolumn{4}{|l|}{ Drug use } \\
\hline Clopidogrel use & $31(100 \%)$ & $87(100 \%)$ & - \\
\hline Aspirin use & $31(100 \%)$ & $87(100 \%)$ & - \\
\hline Statins & $31(100 \%)$ & $87(100 \%)$ & - \\
\hline Heparin & $34(80.95 \%)$ & $61(80.26 \%)$ & 0.9279 \\
\hline B blockers & $30(71.43 \%)$ & $63(82.89 \%)$ & 0.1445 \\
\hline Angiotensin converting enzyme inhibitors & $9(21.43 \%)$ & $24(31.58 \%)$ & 0.2395 \\
\hline \multicolumn{4}{|l|}{ Hematology parameters } \\
\hline White blood corpuscles $\left(\times 10^{9} / \mathrm{L}\right)$ & $7.61 \pm 2.71$ & $7.89 \pm 3.39$ & 0.6212 \\
\hline Red blood corpuscles $\left(\times 10^{12} / \mathrm{L}\right)$ & $4.36 \pm 0.58$ & $4.57 \pm 0.46$ & 0.0473 \\
\hline Platelet $\left(\times 10^{9} / \mathrm{L}\right)$ & $217.41 \pm 81.76$ & $211.86 \pm 54.22$ & 0.6946 \\
\hline Hemoglobin $(\mathrm{g} / \mathrm{L})$ & $128.92 \pm 14.93$ & $138.75 \pm 14.61$ & 0.0110 \\
\hline \multicolumn{4}{|l|}{ Biochemical parameters } \\
\hline Sodium $(\mathrm{mM})$ & $141.10 \pm 5.36$ & $142.11 \pm 2.53$ & 0.3780 \\
\hline Potassium (mM) & $4.05 \pm 0.36$ & $3.96 \pm 0.27$ & 0.2705 \\
\hline Chlorine (mM) & $105.40 \pm 5.00$ & $105.53 \pm 2.61$ & 0.9035 \\
\hline Creatine kinase (U/L) & $61.25(39.83,152.55)$ & $74.4(58.1,107.6)$ & 0.8985 \\
\hline Creatine kinase-MB (U/L) & $8.31(2.84,14.25)$ & $7.9(2.87,12.93)$ & 0.5093 \\
\hline Glucose (mM) & $6.00 \pm 1.87$ & $6.34 \pm 2.08$ & 0.3651 \\
\hline High Density Lipoprotein-C (mM) & $1.09 \pm 0.43$ & $0.99 \pm 0.26$ & 0.1817 \\
\hline Low Density Lipoprotein-C (mM) & $2.70 \pm 1.25$ & $2.52 \pm 1.12$ & 0.4315 \\
\hline Total cholesterol $(\mathrm{mM})$ & $4.13 \pm 1.34$ & $4.06 \pm 1.32$ & 0.7835 \\
\hline Triglycerides (mM) & $1.42 \pm 0.58$ & $1.52 \pm 0.78$ & 0.4334 \\
\hline
\end{tabular}

AA or ADP-induced platelet inhibition rate is indicated by the MA decrease percentage in the curve. Specific equation use to calculate the inhibition rate was as follows: Platelet inhibition rate $(\%)=\left[1-\left(\mathrm{MA}_{\mathrm{p}}-\mathrm{MA}_{\mathrm{Fib}}\right) /\right.$ $\left.\left(\mathrm{MA}_{\text {Thrombin }}-\mathrm{MA}_{\mathrm{Fib}}\right)\right]$ x $100 \%$, where, Map is $\mathrm{MA}_{\mathrm{ADP}}$ or $\mathrm{MA}_{\mathrm{AA}}$. Criteria used for classification was (a) low aspirin reaction, where in case of administration of aspirin at the rate of $1 \mathrm{mM}$ AA induced platelet aggregation inhibition rate $\leq 50 \%$; and (b) low clopidogrel response, where in case of administration of Clopidogrel, $2 \mu \mathrm{M}$ ADP induced platelet aggregation inhibition rate $<30 \%$. 


\section{Coagulation indicators and TEG results}

As expected, the low responder group had a significantly lower ADP $(23.93 \pm 21.93$ $\left.v_{s} 62.15 \pm 20.92\right)$ and $\mathrm{AA}(51.92 \pm 30.17$ vs $89.27 \pm 13.50)$ platelet aggregation inhibition rate $(\mathrm{P}=0.000$ in each case). However, there was no significant difference in other routine coagulation indices between the normal and low responder groups (data not shown). There was no statistical difference in the coronary stenosis distribution $(\mathrm{P}=0.4080)$ and severity (Gensini scores: low responders, $58.83 \pm 41.10$ and normal responder, $62.62 \pm 45.46, \mathrm{P}=0.6733$ ). There was no observed difference in the 2 responder groups regarding the number, length, or diameter of the stent implantation in PCI (data not shown). No significant correlation was found between the responder groups and acute myocardial infarction or unstable angina pectoris $(\mathrm{P}>0.05$ in each case); however, low responders appeared to be negatively correlated with stable angina pectoris classification $(\mathrm{P}<0.05)$.

\section{Multifactor logistic regression analysis}

We examined whether a combination of gender, age, red blood corpuscles, hemoglobin, and clinical classification (each was a dependent variable in univariate analysis) would be a better outcome predictor. As shown in Table 2, age [odds ratio (OR): 6.30; 95\% confidence interval $(95 \% \mathrm{CI}): 1.541-25.762 ; \mathrm{P}=0.0104]$ and clinical classification $(\mathrm{OR}: 4.52 ; 95 \% \mathrm{CI}$ : $1.279-15.962 ; \mathrm{P}=0.0192$ ) were the only independent risk factors in the low responder group.

\begin{tabular}{|c|c|c|c|}
\hline Variable & OR values & $95 \% \mathrm{CI}$ & $\mathrm{P}$ \\
\hline $\mathrm{RBC}$ & 1.11 & $0.244-5.060$ & 0.8917 \\
\hline $\mathrm{Hb}$ & 0.96 & $0.914-1.015$ & 0.1571 \\
\hline Age & 6.30 & $1.541-25.762$ & 0.0104 \\
\hline Gender & 0.23 & $0.038-1.405$ & 0.1119 \\
\hline Clinical classification & 4.52 & $1.279-15.962$ & 0.0192 \\
\hline
\end{tabular}

\section{Ischemic events during follow-up after PCI}

The occurrence of recurrent ischemia during the follow-up occurred in 31 cases $(26.27 \%)$ (in-stent restenosis: 13 cases; unstable angina pectoris requiring hospitalization: 10 cases; acute myocardial infarction: 2 cases; stroke: 4 cases; death: 2 cases). These 31 patients were grouped into the IERG, while the other 87 patients formed the NIERG. IERG occurred significantly more frequently in low responders $(45.24 \%)$ than in normal responders $(15.79 \%)(\mathrm{P}=0.005)$. In comparison, NIERG was more prominent in normal responders $(84.21 \%)$ than in low responders $(54.76 \%)(\mathrm{P}<0.05)$. Inhibition of platelet aggregation rate (ADP and AA) was significantly lower in the IERG $(31.33 \pm 24.91 \%$ and $66.84 \pm 29.35 \%$, respectively) than in NIERG (54.68 \pm $26.63 \%$ and $79.23 \pm 26.28 \%$, respectively) ( $\mathrm{P}=0.000$ for $\mathrm{ADP}$ and 0.0435 for $\mathrm{AA}$, respectively).

\section{Genetic polymorphism of $C Y P 3 A 5 * 3(22893 \mathrm{G}>\mathrm{A}), C Y P 2 C 19 * 2(681 \mathrm{G}>\mathrm{A})$, and $P 2 Y 12 * 1(744 \mathrm{~T}>\mathrm{C})$ and modulation of clopidogrel response}

Three polymorphic forms of each allele were identified (Figure 1 and Table 3). In 
each case, the mutant allele was positively correlated with a significantly lower ADP-induced platelet inhibition rate $(C Y P 2 C 19 * 2$ : wild-type: $55.89 \pm 26.71 \%$; mutant: $43.15 \pm 27.97 \%$, P $=0.0141 ;$ CYP $3 A 5 * 3$ : wild-type: $52.89 \pm 26.49 \%$; mutant: $41.98 \pm 29.33 \%, \mathrm{P}=0.038$; and $P 2 Y 12 * 1$ : wild-type: $52.19 \pm 28.58 \%$; mutant: $38.74 \pm 24.36 \%$; $=0.0199)$.

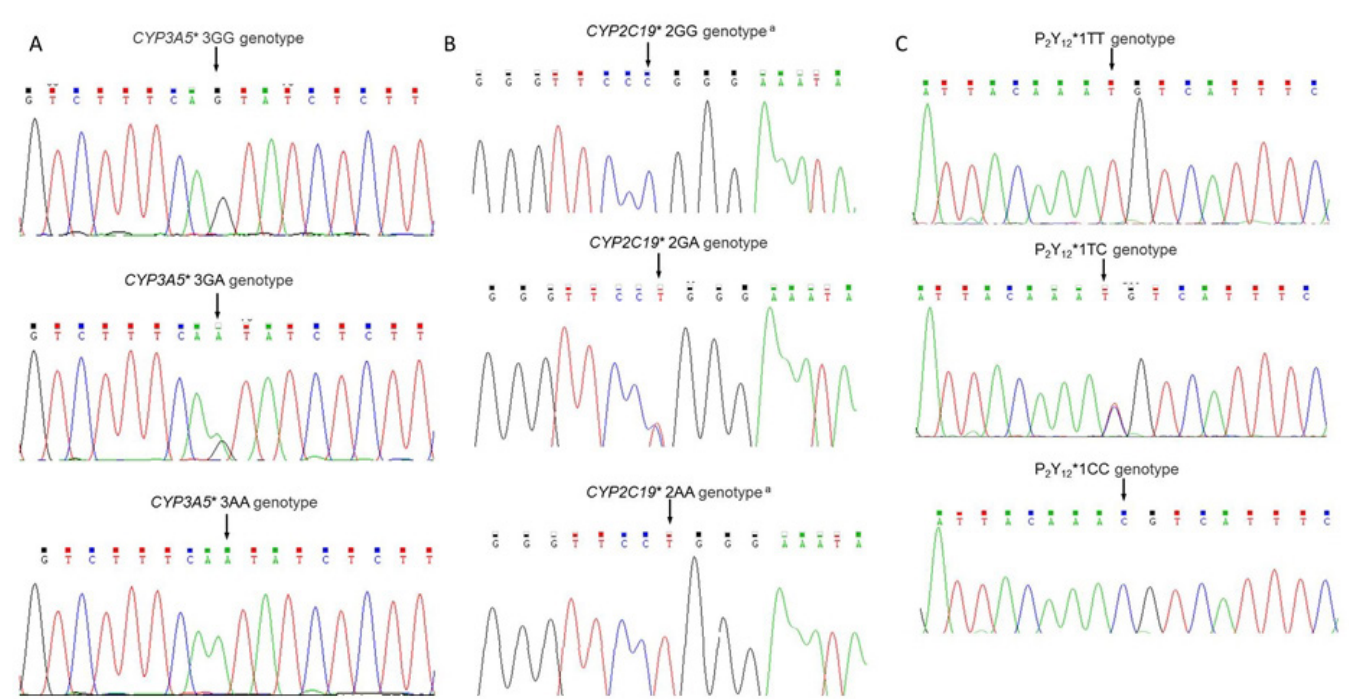

Figure 1. Representative chromatograms of the different polymorphic alleles for $C Y P 3 A 5 * 3$ (A), CYP $2 C 19 * 2$ (B), and $P 2 Y 12 * 1(\mathbf{C})$.

Table 3. Genotype distribution of $C Y P 3 A 5 * 3, C Y P 2 C 19 * 2, P 2 Y 12 * 1$ and comparison of detection results between wild type and mutant TEG. PCR product was sequenced and analyzed. Representative expression of the different polymorphic alleles for each of three aforementioned genes is shown on Table.

\begin{tabular}{|c|c|c|c|c|c|c|}
\hline \multirow[t]{2}{*}{ Loci } & \multirow{2}{*}{$\begin{array}{c}\text { Wild-type } \\
\text { (homozygous) }\end{array}$} & \multirow{2}{*}{$\begin{array}{c}\text { Mutant } \\
\text { (heterozygous) }\end{array}$} & \multirow{2}{*}{$\begin{array}{c}\text { Mutant } \\
\text { (homozygous) }\end{array}$} & \multicolumn{2}{|c|}{ ADP-induced platelet inhibition rate (\%) } & \multirow[t]{2}{*}{$\mathrm{P}$} \\
\hline & & & & Wild-type & Mutant & \\
\hline$C Y P 3 A 5 * 3$ & GG $71(0.602)$ & GA $39(0.331)$ & AA $8(0.607)$ & $52.89 \pm 26.49$ & $41.98 \pm 29.33$ & 0.038 \\
\hline CYP $2 C 19 * 2$ & GG $50(0.424)$ & GA $60(50.9)$ & AA $8(0.067)$ & $55.89 \pm 26.71$ & $43.15 \pm 27.97$ & 0.0141 \\
\hline$P 2 Y 12 * 1$ & TT $86(0.729)$ & $\mathrm{TC}(0.229)$ & $\mathrm{CC}(0.042)$ & $52.19 \pm 28.58$ & $38.74 \pm 24.36$ & 0.0199 \\
\hline
\end{tabular}

The CYP $3 A 5 * 3(22893 \mathrm{G}>\mathrm{A})$ frequency of the wild-type GG genotype in NIERG was $70.12 \%$, which was significantly higher than the $32.26 \%$ in the IERG. In comparison, the GA genotype in patients with IERG was significantly lower than those with the GG genotype $(\mathrm{P}<0.05)$; the frequency of the A allele in the IERG was $38.71 \%$, which was significantly higher than blood transfusion recurrence of $17.82 \%$ in this group. Patients carrying the A allele showed an incidence rate of ischemic events of $43.64 \%$, which was much higher than in those with the $\mathrm{G}$ allele at $20.99 \%(\mathrm{P}<0.05)$.

The CYP2C19*2 (681G>A) frequency of the GG genotype in NIERG was $51.72 \%$, which was significantly higher than the $16.13 \%$ in the IERG; in patients with GA and AA genotypes, the risks of ischemic events were 35 and $62.5 \%$, which were higher than those for 
patients with the GG genotype $(10 \%)(\mathrm{P}<0.05)$; the frequency of the A allele in the IERG was $50 \%$, which was significantly higher than that in the NIERG.

Finally, for $P 2 Y 12 * 1(744 \mathrm{~T}>\mathrm{C})$, the frequencies of the TT genotype in the IERG and NIERG were 72.41 and $74.19 \%$, respectively, indicating that the chance of recurrence of ischemic events for patients with the TT, TC, and CC genotypes were 26.74, 25.93, and 20\%, respectively $(\mathrm{P}>0.05)$; the frequencies of the $\mathrm{C}$ allele in the IERG and NIERG were 14.52 and $16.09 \%$, and the $\mathrm{T}$ allele and $\mathrm{C}$ allele in patients with ischemic events showed frequencies of 26.63 and $24.32 \%$, respectively $(\mathrm{P}>0.05)$.

\section{DISCUSSION}

In this study, the TEG test results showed that patients with poor response to antiplatelet drugs had a significantly higher risk of ischemic events occurring during follow-up compared to normal responders. Our results agree with those of previous studies regarding the benefit of measuring outcome criterion after clopidogrel treatment (Nguyen et al., 2005). However, our logistic regression analysis showed that age and severity of coronary heart disease were independent risk factors for the impact of platelet inhibition, which was not statistically significant between the NIERG and IERG groups, suggesting that other factors affect the rate of platelet inhibition. Thus, TEG platelet mapping can only partially predict the efficacy of antiplatelet drugs.

Clopidogrel differences can be multifaceted and are mainly related to gene polymorphisms (Gutliikonda et al., 2005), the degree of disease risk (Soffer et al., 2003), hyperinsulinism or insulin resistance (Kernan and Inzucchi, 2004), patients with poor compliance, drug dose (Montalescot et al., 2006), individual differences in drug absorption and clearing the active metabolite, drug interactions (Gurbel et al., 2005b), and platelet bypass via activation.

Some limitations of our study design were that the sample size was small and that we only screened polymorphisms of 3 genes. Future studies will focus on incorporating $A B C B-1$ and $C Y P 3 A 4$. The current study showed that the presence of the $C Y P 3 A 5 * 3(22893 \mathrm{G}>\mathrm{A})$ or CYP2C19*2 $(681 \mathrm{G}>\mathrm{A})$ mutant allele in patients after PCI was more likely to be associated with re-ischemic events, while the impact of the $P 2 Y 12 * 1(744 \mathrm{~T}>\mathrm{C})$ polymorphism on the recurrence of ischemic events was not significant. The risk of postoperative recurrence of ischemic events was significantly higher when multi-locus mutations were present.

With the advent of personalized genomic medicine, coronary heart disease patients undergoing PCI or long-term use of clopidogrel should be screened for genetic polymorphisms. For cases in which polymorphisms are present, individualized treatment of patients such as increasing clopidogrel dose or selecting other ADP receptor antagonists (e.g., prasugrel, Kangeleiluo) in conjunction with GP IIb/IIIa receptor antagonists and other antiplatelet drugs should be considered.

\section{ACKNOWLEDGMENTS}

Research supported by General Program of the 309th Hospital of People's Liberation Army (\#2013MS-018). We thank Dr. Xuexiong Wu, Director of Research Institute of Tuberculosis, the 309th Hospital of PLA, Dr. Junxian Zhang and Dr. Yourong Yang for providing assistance in experimental procedures. We also thank Dr. Chengbin Wang, Director of Clinical Laboratory, General Hospital of PLA, Ms. Li Yang, and Mr. Quancheng Xia in guiding quality control. 


\section{REFERENCES}

Angiolillo DJ, Fernandez-Ortiz A, Bernardo E, Ramírez C, et al. (2005). Lack of association between the P2Y12 receptor gene polymorphism and platelet response to clopidogrel in patients with coronary artery disease. Thromb. Res. 116: 491-497.

Angiolillo DJ, Fernandez-Ortiz A, Bernardo E, Alfonso F, et al. (2007). Variability in individual responsiveness to clopidogrel: Clinical implications, management, and future perspectives. J. Am. Coll. Cardiol. 49: 1505-1516.

Balram C, Zhou Q, Cheung YB and Lee EJ (2003). CYP3A5*3 and *6 single nucleotide polymorphisms in three distinct Asian populations. Eur. J. Clin. Pharmacol. 59: 123-126.

Barsky AA and Arora RR (2006). Clopidogrel resistance: myth or reality. J. Cardiovasc. Pharmacol. Ther. 11: 47-53.

Bliden KP, Tantry U, Guyer K, Hayes K, et al. (2005). High platelet reactivity is a risk factor for post-discharge ischemic complications following elective coronary stenting. J. Am. Coll. Cardio. 47: 45B.

Camilleri E, Jacquin L, Paganelli F and Bonello L (2011). Personalized antiplatelet therapy: review of the latest clinical evidence. Curr. Cardiol. Rep. 13: 296-302.

Chen H, Yan W and Wu XY (2010). Relationships of blood stasis syndrome, CYP2C19 gene polymorphism with clopidogrel resistance and post-PCI prognosis. Zhongguo Zhong Xi Yi Jie He Za Zhi. 30: 1245-1249.

Evans WE and McLeod HL (2003). Pharmacogenomics - drug disposition, drug targets, and side effects. N. Engl. J. Med. 348: 538-549.

Fitzgerald DJ and Maree A (2007). Aspirin and clopidogrel resistance. Hematology 1: 114-120.

Fontana P, Dupont A, Gandrille S, Bachelot-Loza C, et al. (2003). Adenosine diphosphate-induced platelet aggregation is associated with P2Y12 gene sequence variations in healthy subjects. Circulation 108: 989-995.

Gurbel PA, Bliden KP, Guyer K, Cho PW, et al. (2005a). Platelet reactivity in patients and recurrent events poststenting: results of the prepare post-stenting study. Am. Coll. Cardiol. 4: 1820-1826.

Gurbel PA, Bliden KP, Zaman KA, Yoho JA, et al. (2005b). Clopidogrel loading the clopidogrel loading with eptifibatide to arrest the reactivity of platelets: results of the clopidogrel with eptifibatide to arrest the reactivity of platelets: (CLEAR PLATEIETS) study. Circulation 111: 1153-1159.

Gutliikonda S, Lev EI and Klieiman NS (2005). Resistance to antiplatelet therapy. Curr. Cardiol. Rep. 7: 242-248.

Jeong YH, Kim IS, Park Y, Kang MK, et al. (2010). Carriage of cytochrome 2C19 polymorphism is associated with risk of high post-treatment platelet reactivity on high maintenance-dose clopidogrel of $150 \mathrm{mg} /$ day: results of the ACCELDOUBLE (Accelerated Platelet Inhibition by a Double Dose of Clopidogrel According to Gene Polymorphism) study. JACC Cardiovasc Interv. 3: 731-741.

Kernan WN and Inzucchi SE (2004). Type 2 diabetes mellitus and insulin resistance: stroke prevention and management. Curr. Treat. Options Neurol. 6: 443-450.

Kuehl P, Zhang J, Lin Y, Lamba J, et al. (2001). Sequence diversity in CYP3A promoters and characterization of the genetic basis of polymorphic CYP3A5 expression. Nat. Genet. 27: 383-391.

Mega JL, Close SL, Wiviott SD, Shen L, et al. (2009). Cytochrome p-450 polymorphisms and response to clopidogrel. $N$. Engl. J. Med. 360: 354-62.

Montalescot G, Sideris G, Meuleman C, Bal-dit-Sollier C, et al. (2006). A randomized comparison of high clopidogrel loading doses in patients with non-ST-segment elevation acute coronary syndromes: the ALBION (Assessment of the Best Loading Dose of Clopidogrel to Blunt Platelet Activation, Inflammation and Ongoing Necrosis) trial. J. Am. Coll. Cardiol. 48: 931-938.

Nguyen TA, Diodati JG and Pharand C (2005). Resistance to clopidogrel: a review of the evidence. J. Am. Coll. Cardiol. 45: 1157-1164.

Pettersen AA, Arnesen H, Opstad TB and Seljeflot I (2011). The influence of CYP 2C19*2 polymorphism on platelet function testing during single antiplatelet treatment with clopidogrel. Thromb. J. 9: 4.

Sibbing D, Koch W, Gebhard D, Schuster T, et al. (2010). Cytochrome 2C19*17 allelic variant, platelet aggregation, bleeding events, and stent thrombosis in clopidogrel-treated patients with coronary stent placement. Circulation 121: 512-518.

Soffer D, Moussa I, Harjai KJ, Boura JA, et al. (2003). Impact of angina class on inhibition of platelet aggregation following clopidogrel loading in patients undergoing coronary intervention: do we need more aggressive dosing regimens in unstable angina? Catheter Cardiovasc. Interv. 59: 21-25.

Sugunaraj JP, Palaniswamy C, Selvaraj DR, et al. (2010). Clopidogrel resistance. Am. J. Ther. 17: 210-215.

Tantry US, Bliden KP and Gurbel PA (2005). Overestimation of platelet aspirin resistance: detection by TEG platelet mapping and validation by conventional aggregometry using arachidonic acid stimulation. J. Am. Coll. Cardiol. 46: 1705-1709.

Wang TH, Bhatt DL and Topol EJ (2006). Aspirin and clopidogrel resistance: an emerging clinical entity. Eur. Heart J. 27: 647-654.

Xie HG, Wood AJ, Kim RB, Stein CM, et al. (2004). Genetic variability in CYP3A5 and its possible consequences. Pharmacogenomics 5: 243-272. 